\title{
Fachkommunikation im Recht unter Berücksichtigung der Mehrfachadressierung
}

\section{Einleitung}

In der Fachsprachenforschung ${ }^{1}$ sind nach der sogenannten kommunikativ-pragmatischen Wende Anfang der 70er Jahre des 20. Jahrhunderts ${ }^{2}$ die Gelingensbedingungen von (Fach)Kommunikation als Forschungsgegenstand mehr und mehr ins Visier geraten. ,Fachkommunikation' soll hier in Anlehnung an die Definitionen von Hoffmann ${ }^{3}$ und Picht $^{4}$ verstanden werden. Hoffmann fokussiert in seiner Definition auf die Exteriorisierung und Interiorisierung von Kenntnissystemen und den dabei sich abspielenden kognitiven Prozessen, während Picht seine Definition stärker auf die bei der Fachkommunikation aktualisierten Mittel - sprachliche oder andere semiotische Mittel - und das fachkommunikative Handeln ausrichtet. Beide Definitionen sind für die folgenden Ausführungen höchst relevant.

Für die Fachkommunikation im Allgemeinen gelten folgende Voraussetzungen:

- Wahre Mitteilung kann nur unter "Gleichgesinnten, Gleichdenkenden" stattfinden, meinte bereits Novalis ${ }^{5}$, und kann mutatis mutandis auf die Kommunikationsteilnehmer in der Fachkommunikation angewandt werden.

\footnotetext{
${ }^{1}$ Vgl. hierzu das zentrale Werk von LOTHAR HOFFMANN/HARTWIG KALVERKÄMPER/ HERBERT ERNST WIEGAND (Hrsg.), Fachsprachen - Languages for Special Purposes. Ein internationales Handbuch zur Fachsprachenforschung und Terminologiewissenschaft - An International Handbook of Special Languages and Terminology Research. 1. Halbbd. Berlin 1998.

2 GerHARd HeLbiG, Entwicklung der Sprachwissenschaft seit 1970. 2. Aufl, Leipzig 1986, 13.

${ }^{3}$ LOTHAR HOFFMANN, Fachwissen und Fachkommunikation. Zur Dialektik von Systematik und Linearität in den Fachsprachen, in: THEO BUNGARTEN, Fachsprachentheorie. Bd. 2: Konzeptionen und theoretische Richtungen. Tostedt 1993, 595 ff., 614.

${ }^{4}$ HeRIBERT PICHT, Fachkommunikation - Fachsprache, in: GERHARD BUdiN, Multilingualism in specialist communication: Proceedings of the 10th European LSP Symposium. Vienna 29. Aug.-1. Sept. 1995. Bd. 1., Wien 1996, 27 ff., 44.

${ }^{5}$ Novalis, Novalis' Werke. Dritter Teil. Fragmente 1. Herausgegeben von HERMANN FRIEDEMANN. Berlin 1921, 74.
} 
- Das Decodieren sprachlicher Information kann nur gelingen, wenn ausreichendes Wissen beim Rezipienten bzw. Adressaten vorhanden ist, ,Wissen' hier verstanden als zumindest „allgemeine Kenntnis enzyklopädischer Fakten."6

Für die Fachkommunikation im Recht kommen noch folgende zwei Voraussetzungen hinzu:

- Sprache ist für das Recht unabdingbar, denn ohne Sprache ist das Recht nicht kommunizierbar, wie schon Vanderlinden festgestellt hat. ${ }^{7}$

- Das Diktum, dass ein Rechtstext gleichzeitig verständlich, aber auch unmissverständlich sein soll(te). ${ }^{8}$

\section{Fragestellungen und theoretische Lösungsansätze}

Aus obigen Voraussetzungen ergeben sich folgende Fragestellungen, denen hier genauer nachgegangen werden soll:

1. Welche Strategien verwendet der Absender in der Fachkommunikation bei Berücksichtigung der Mehrfachadressierung als "Gleichgesinnte" und "Nicht-Gleichgesinnte”, um der Anforderung von gleichzeitiger Verständlichkeit und Unmissverständlichkeit im Recht gerecht zu werden?

2. Wie kann dem "Nicht-Gleichgesinnten" - zumindest - ein Mindestmaß an Fachwissen/Rechtswissen vermittelt werden?

3. Wie kann und sollte sich der Absender einer Mitteilung auf seine(n) Adressaten einstellen, um sicher zu sein, dass seine Botschaft auch ankommt?

In der antiken Rhetorik hatte Hermagoras von Temnos (200 v. Chr.) bereits auf die verschiedenen Faktoren des quis quid quando ubi cur quem ad modum quibus adminiculis (wer, was, wann, wo, weshalb, mit welchen Mitteln) hingewiesen, was im 20. Jahrhundert in der bekannten Lass-

${ }^{6}$ INGO WARNKE, Transferwissenschaftliche Aspekte der Schwerverständlichkeit deutscher Gesetzestexte, in: SIGURD WICHTER/GERD ANTOS, Wissenstransfer zwischen Experten und Laien. Umriss einer Transferwissenschaft. Frankfurt am Main 2001, 193 ff., 201.

7 JaCQues VANDERLINDEN, Langue et Droit, in: ERIK JAYME, Langue et Droit. XVe Congrès International de Droit Comparé, Bristol 1998, Collection des rapports. Brüssel 1999, 65 ff., 73. Ähnlich auch RUDOLF HAAS, Recht und Sprache, in: DERS., Recht und Sprache. Festschrift zum 10jährigen Bestehen der Deutschen Anwaltsakademie. München 1989, 39 ff., 41.

${ }^{8}$ WOLFGANG KLEIN, Sprache des Rechts. Vermitteln, Verstehen, Verwechseln, in: Zeitschrift für Literaturwissenschaft und Linguistik 118 (2000), $7 \mathrm{ff.}, 9$. 
well'schen Formel ${ }^{9}$ "wer sagt was, auf welchem Weg, zu wem, mit welcher Wirkung?" wieder aufgegriffen wurde. Zwar war diese Formel ursprünglich für den Bereich der Massenkommunikation konzipiert, ist aber von anderen Disziplinen, einschließlich der (angewandten) Sprachwissenschaft und der sich zwischenzeitlich als eigenständige Disziplin daraus abgespaltenen Fachsprachenforschung für ihre Zielsetzung vereinnahmt worden. Weitere Fragestellungen sind zu den ursprünglichen hinzugefügt worden, unter anderem die der Zielsetzung dessen, was gesagt bzw. geschrieben wird. Somit liegen hier Anknüpfungspunkte an eine allgemeine Kommunikationstheorie vor, die für vorliegenden Untersuchungszweck nützlich sein kann.

Daneben ist aber auch die Fachsprachen-Hermeneutik ${ }^{10} \mathrm{zu}$ berücksichtigen, auf deren Bedeutung etwa Kalverkämper hingewiesen hat, ${ }^{11}$ deren Zielsetzung mehr ist als nur ein Ersetzen oder Vermeiden von Fachwörtern (Termini), sondern eben darauf abzielt, das vom Textautor/Sender im Voraus zu berücksichtigende Wissensgefälle durch $u$. a. Umschreibungen (Paraphrasen) zu vermindern.

Bei Zugrundelegen solcher theoretischen Ansätze wären dann für die Fachkommunikation im Recht folgende Parameter zu berücksichtigen: (1) die beteiligten Kommunikationsteilnehmer, (2) die intendierte Botschaft des Senders und seine Berücksichtigung der Adressaten und (3) die Rezeption der Botschaft bei dem/den Adressaten, (4) eventueller "Lärm" und (5) das jeweilige Vorwissen beim Adressaten.

Die beteiligten Kommunikationsteilnehmer werden hier als Sender bzw. Adressat ${ }^{12}$ bezeichnet, die intendierte Botschaft soll für den Zweck dieses Beitrags zwei zentrale Typen von Rechtstexten umfassen, während die Termini - im Sinne der DIN $2342^{13}$ - bei unterschiedlichem Wissensstand der Adressaten die Kommunikation als sogenannter "Lärm" be- oder sogar verhindern können.

Die schriftlichen Rechtstexte sind aufgrund verschiedener textexterner und -interner Kriterien abgrenzbar und umfassen einer üblichen

${ }^{9}$ HAROLD D. LASSWEll, The Structure and Function of Communication in Society, in: LXMAN BRYSON, The Communication of Ideas. A series of addresses. New York 1948, $37 \mathrm{ff.}$ 37.

10 Siehe auch INGRID SIMONNÆS, Zur Hermeneutik als Verstehenshilfe bei Rechtstexten, in: LSP \& Professional Communication. An International Journal (Formerly Unesco AlsedLSP Newsletter) 1 (2001), 78 ff., 100.

${ }^{11}$ HARTWIG KALVERKÄMPER, Fachexterne Kommunikation als Maßstab einer Fachsprachen-Hermeneutik: Verständlichkeit kernphysikalischer Fakten in spanischen Zeitungstexten, in: DERS., Fachsprachen in der Romania. Tübingen 1988, $151 \mathrm{ff} ., 161 \mathrm{f}$.

12 Zur Differenzierung der Adressaten von generellen Normen des Rechts vgl. HANS KELSEN, Allgemeine Theorie der Normen. Wien 1979, $40 \mathrm{f}$.

13 DIN 2342 TEIL 1, Begriffe der Terminologielehre. Grundbegriffe. Normenausschuß Terminologie (NAT). DIN Deutsches Institut für Normung e. V. Berlin 1992, 3. 
Gliederung zufolge normative und rechtsanwendende Texte. ${ }^{14}$ Die oben angeführten Fragestellungen sollen im Weiteren anhand einer Gruppe solcher schriftlichen Rechtstexte getestet werden; andere Gruppen von Rechtstexten bleiben für diesen Beitrag unberücksichtigt.

\section{Beschreibung des Analysematerials}

Unter II. wurde darauf hingewiesen, dass sowohl normative Texte, also Texte, die Recht setzen, wie etwa formelle Gesetze, als auch rechtsanwendende Texte, $z u$ denen gerichtliche Entscheidungen gehören, als "Botschaft" im kommunikationstheoretischen Sinne zu sehen wären.

Bei der empirischen Analyse wurde von zwei straf- und zwei zivilrechtlichen Urteilen von der ersten bis zur letzten Instanz im Umfang von insgesamt ca. 90 Seiten ausgegangen. ${ }^{15}$ Die Auswahl der Urteile wurde vor dem Hintergrund des Bekanntheitsgrads beim Leser getroffen: "Man" weiß, dass Diebstahl und Mord verboten (und mit Strafe belegt) sind (siehe die Zehn Gebote, deren Kenntnis man - in unserer Kultur - voraussetzen darf) bzw. "man" weiß, dass man in bestimmten Fällen, z. B. nach einem Verkehrsunfall, zu Schadenersatz verpflichtet sein kann. Die Auswertung der Urteile hat sich im Wesentlichen auf den Tenor (einschließlich der dort aufgeführten gesetzlichen Vorschriften) und die Entscheidungsgründe beschränkt, da der Richter im letzteren Teiltext ${ }^{16}$ eines Urteils darzulegen hat, warum er einen bestimmten Einzelfall unter bestimmte Gesetzesbestimmungen subsumiert.

Urteile lassen sich aufgrund textexterner Merkmale, wie textexterner Funktion, Kommunikationssituation und Verhältnis zwischen Sender und Adressat, einer eigenen identifizierbaren Textsorte zuordnen, die in verschiedene Teiltexte zerfällt. Die textexterne Funktion ist hier - wie bei anderen gerichtlichen Entscheidungen ${ }^{17}$ - einerseits das Informieren, andererseits das Anweisen. Das Informieren im Teiltext Urteilsformel

${ }^{14}$ Eine hiervon etwas abweichende Bezeichnung findet sich bei PETER KÜHN, Juristische Fachtexte, in: GERHARD HELBIG/LUTZ GÖTZE/GERT HENRICI/HANS-JÜRGEN KRUMM (Hrsg.), Deutsch als Fremdsprache. Ein internationales Handbuch. Berlin 2001, 582 ff., 586.

${ }^{15}$ Ausführlicher hierzu in INGRID SIMONNAES, Verstehensprobleme bei Fachtexten. $\mathrm{Zu} B e-$ griffssystemen und Paraphrasen als Visualisierungs- bzw. Verbalisierungsinstrumente in der Kommunikation zwischen Fachmann und Laien. Eine Untersuchung anhand gerichtlicher Entscheidungen. Diss. Bergen 2003.

${ }^{16}$ Zur Defirition von ,Teiltext' vgl. Elisabeth GÜLICH/WOLFGANG RAIBLE, TextsortenProbleme, in: Hugo Moser/Hans EgGers/JOHANNES ERBEN/Odo LeYs/Hans NeuMANN (Hrsg.), Linguistische Probleme der Textanalyse. Jahrbuch 1973. Düsseldorf 1975, 144 ff., 146 .

${ }^{17}$ Zur Gliederung von gerichtlichen Entscheidungen siehe u. a. KURT SCHELLHAMMER, Die Arbeitsmethode des Zivilrichters. 12. Aufl., Heidelberg 1997, 297. 
kann bei Strafverfahren beispielsweise darin bestehen, den Angeklagten davon in Kenntnis zu setzen, dass er zu einer Freiheitsstrafe von $x$ Jahren verurteilt worden ist. In einem Zivilverfahren wird die unterlegene Partei darüber informiert, dass sie mit ihrer Klage nicht erfolgreich gewesen ist. Bei erstinstanzlichen Urteilen wird aber auch die nächste Instanz, im Falle, dass Berufung oder Revision eingelegt wird, informiert. Der Zweck eines Urteils ist schließlich auch das Anweisen, z. B. bei Strafverfahren das Anweisen an die zuständige Vollzugsbehörde, den Angeklagten entsprechend lange in Haft zu nehmen, sobald ein rechtskräftiges Urteil vorliegt, oder das Anweisen an die unterlegene Partei in einem Zivilverfahren, für die Prozesskosten aufkommen zu müssen.

Als zentrales textexternes Merkmal sehe ich die Kommunikationssituation, das Sender-Adressaten-Verhältnis, beim Verkünden des Urteils durch den Richter an den Rechtsunterworfenen, also durch den Fachmann an den Laien, bzw. das Lesen des Urteils. Außerdem ist die $\mathrm{Zu}$ sammensetzung des erkennenden Gerichts zu berücksichtigen: In den Fällen, in denen ehrenamtliche Richter, also juristisch nicht vorgebildete Richter, mitwirken, ist in der Kommunikationssituation ebenfalls ein Fachmann-Laien-Verhältnis gegeben. Es ist naheliegend anzunehmen, dass sich dies auch sprachlich manifestiert, was durch die Untersuchung über die Verwendung von Termini bzw. Paraphrasen eben empirisch nachgewiesen werden soll.

Die rechtssetzenden Texte sind hierbei insofern von Interesse, als die Urteilstexte auf sie verweisen: bei Strafurteilen im Tenor, bei zivilrechtlichen Urteilen allerdings erst, nachdem die Anspruchsgrundlage im Teiltext Entscheidungsgründe konkretisiert worden ist.

Die Urteile wurden daraufhin untersucht, wie und wo Termini, die bekanntlich eine Komprimierung von Fachwissen darstellen und der sprachökonomischen Kommunikation unter Fachleuten dienen (sollen), durch Paraphrasen ersetzt worden sind. Diese Zielsetzung ergab sich aus folgenden drei Hypothesen:

- Der Sender (= Richter) wendet mit Blick auf die Mehrfachadressierung bei der Abfassung des Urteils eine Doppelstrategie an. Er muss darauf achten, dass beim Rechtsunterworfenen, einschließlich eventueller ehrenamtlicher Richter, ein Verständnis der Entscheidung gesichert ist, die Entscheidung also verständlich ist. Gleichzeitig muss er aber darauf achten, dass seine Entscheidung einer rechtlichen Nachprüfung in der nächsten Instanz standhält, also unmissverständlich ist. Aus der Sicht der Kommunikationstheorie gesehen stehen dem Sender (= Richter) also zwei Adressaten gegenüber, A1 und A2. 
- Bei den unterschiedlichen Adressaten trägt der Gebrauch von Termini bzw. deren Paraphrasen zur besseren Verständlichkeit bei.

- Der Gebrauch von Paraphrasen nimmt bei letztinstanzlichen Urteilen $a b$.

Gegenstand dieser Analyse sind also deutsche straf- und zivilrechtliche Urteile bzw. Beschlüsse mit ihrem Bezug auf die jeweils relevanten Gesetzestexte, wobei beide aufgrund textexterner und -interner Merkmale als eigene Fachtextsorten ${ }^{18}$ betrachtet werden.

\section{Zentrale Definitionen}

Eine ausführliche Diskussion über die zentralen Begriffe ,Terminus', ,Paraphrase' und ,Verständlichkeit' würde den Rahmen dieses Beitrags sprengen. Daher folgt hier nur eine kurze Diskussion.

- Die Auffassungen darüber, was unter ,Terminus' zu verstehen ist, sind in der Fachliteratur konträr. ${ }^{19}$,Terminus' wird in der diesem Beitrag zugrunde liegenden Untersuchung gemäß der Definition der DIN 2342 gesehen, wonach ein ,Terminus' aus einem Begriff und einer Benennung als Element einer Terminologie besteht. Diese Definition steht im Gegensatz zur ISO 108720, nach der als ,term' nur die sprachliche Ausdrucksseite („verbal designation") gesehen wird. Beispiel: Im Schuldrecht ist, Vertrag' ein Terminus und umfasst $u$. a. Kauf-, Miet- und Werkverträge.

- Bei ,Paraphrase' wird die Definition von ,pragmatischer Paraphrase' sensu Martin ${ }^{21}$ zugrunde gelegt, wobei folgende Abgrenzung vorgenommen wird: ,Paraphrase': unidirektionale Wiederholung mit anderen sprachlichen Zeichen bei gleichzeitigem Wechsel von Begriffsebene auf ontische Ebene. Es geschieht also ein "Heruntersteigen" von der Ebene der abstrakten Gesetzesbegriffe auf die Ebene der Wirklichkeit, den konkreten Einzelfall. Beispiel: Die abstrakte Sache aus § 242 StGB (Diebstahl) wird im konkreten Einzelfall zum Rind, wobei man wissen muss, dass Rinder zur Gattung der Tiere gehören

18 WERNER WOLSKI, Fachtextsorten und andere Textklassen: Probleme ihrer Bestimmung, Abgrenzung und Einteilung, in: HOFFMANN/KALVERKÄMPER/WIEGAND, Fachsprachen (Fn. 1), 457 ff., $460 \mathrm{ff}$.

19 Christer Laurén/JOHAN MYKIng/Heribert PiCht, Terminologie unter der Lupe. Wien $1998,221 \mathrm{ff}$.

20 ISO 1087-1, Terminology work - Vocabulary. Theory and application. Genf 2000.

${ }^{21}$ ROBERT MARTIN, Inférence, Antonymie et Paraphrase, Eléments pour une théorie sémantique. Paris 1976,118 
(Weltwissen), Tiere jedoch nicht Sachen im Sinne des Gesetzes sind, aber auf sie dennoch "die für Sachen geltenden Vorschriften entsprechend anzuwenden [sind], soweit nicht etwas anderes bestimmt ist" (\$ 90a BGB) (Fachwissen). Da keine andere Bestimmung besteht, kann antithetisch geschlussfolgert werden, dass Tiere gesetzlich wie Sachen zu sehen sind. Die "Gleichung" zwischen den sprachlichen Ausdrücken ,bewegliche Sache ${ }^{\prime}=$,Rind' lässt sich aber nur unidirektional lesen, da ein Rind zwar eine bewegliche Sache ist (im Sinne des Gesetzes), nicht aber jede bewegliche Sache ein Rind ist.

- ,Verständlichkeit' wird als kommunikative Qualität einer in einem Terminus verdichteten Textstelle bei Berücksichtigung des situativen Kontextes gesehen, in dem sich ein Sender und unterschiedliche Adressaten gegenüberstehen. Ein Gelingen des Hinüberbringens der ,Botschaft', d. h. dass die Botschaft verständlich ist, mithin verstanden wird, ist nur bei Vorhandensein eines gemeinsamen ,Kodes', bzw. zumindest Teilen davon, bei Sender und Adressat möglich wie im vereinfachten Kommunikationsmodell (siehe Abb. 1).

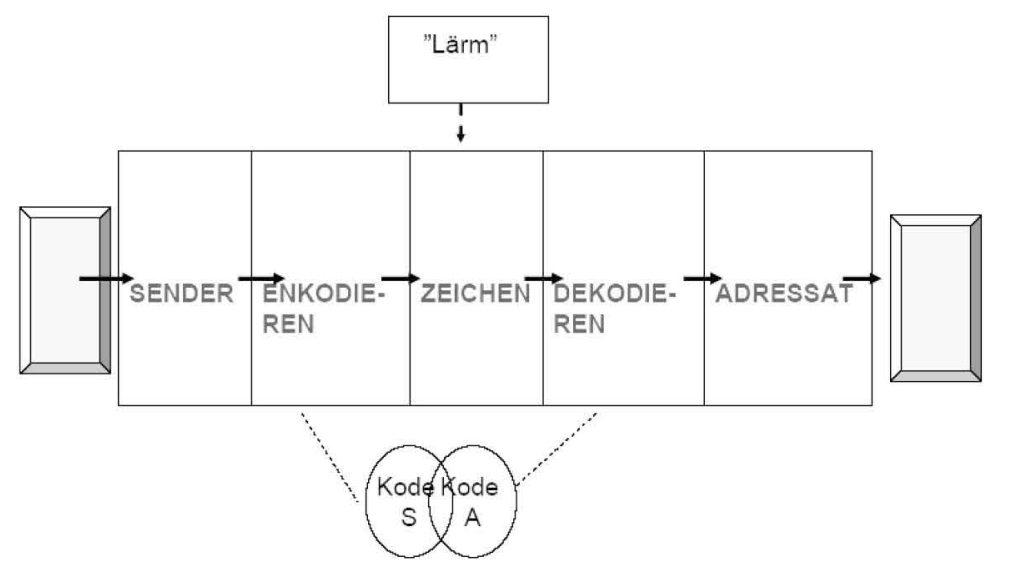

Abb. 1: Vereinfachtes Kommunikationsmodell

In der Fachkommunikation reicht allerdings das gemeinsame Sprachwissen nicht aus. Es kommt noch das Erfordernis des Fachwissens hin$\mathrm{zu}$, wie dies später in Abb. 6 gezeigt wird. Bei Rechtstexten gilt meines 
Erachtens daher mit Recht die Behauptung von Duve/Weirich ${ }^{22}$, dass das Verstehen solcher Texte in erster Linie kein sprachliches Problem ist, sondern im fehlenden Vorverständnis (Gadamer) ${ }^{23}$ begründet werden kann. Diese Auffassung steht auch im Einklang mit den Befunden der kognitiven Linguistik, ${ }^{24}$ wonach die Wichtigkeit der beim Rezipienten vorhandenen Wissensstrukturen hervorgehoben und der Verstehensprozess als eine Interaktion zwischen vorgegebener Textstruktur und der beim Rezipienten vorhandenen Kognitionsstruktur gesehen wird. ${ }^{25}$

Als ,intendierte Botschaft' gelten hier die begriffliche Ebene, die wir in den Urteilen mit Bezug auf das geltende Recht, Gesetze und Dogmatik, finden, und die ontische Ebene, die Ebene des Sachverhalts, wobei beide Ebenen aufeinander zu beziehen sind.

\section{Lösungsansätze in praxi}

Wenn nun in einer konkreten Kommunikationssituation eben keine Gleichdenkenden da sind - was ja der Fall ist, wenn sich Sender und Adressat als Fachmann bzw. Laie gegenüberstehen -, wie kann dann eine Brücke gebaut werden, welche Hilfsmittel können eingesetzt werden, um ein Verstehen zu sichern? Was geschieht, wenn der Sender in der Kommunikationssituation zwei unterschiedliche Adressaten (A1 und A2) berücksichtigen muss, er also eine sogenannte Bringschuld ${ }^{26}$ dem Laien (A2) gegenüber hat, wie dies laut der ersten Hypothese der Fall ist?

Zur Kennzeichnung der Adressaten wurde das heute allgemein akzeptierte Modell des gleitenden Übergangs zwischen Fachsprache und Gemeinsprache benutzt, bei dem die Adressaten einmal als fachintern,

22 HANS ERNST DUVE/HANS-ARMIN WEIRICH, Die Verständigung zwischen dem Bürger und den Juristen kann verbessert werden, in: INGULF RADTKE, Die Sprache des Rechts und der Verwaltung. Stuttgart 1981, $119 \mathrm{ff} ., 121 \mathrm{f}$.

${ }^{23}$ HANS-GeORG GADAMER, Vom Zirkel des Verstehens, in: GÜNTHER NESKE, Martin Heidegger zum siebzigsten Geburtstag. Festschrift. Pfullingen 1959, 24 ff., 30.

${ }^{24}$ Vgl. hierzu MONIKA SCHWARZ, Einführung in die kognitive Linguistik. 2. Aufl., Tübingen 1996, Kap. 2.

${ }^{25}$ Vgl. hierzu URSUla CHRISTMANN/NORBERT GROEBEN, Textverstehen, Textverständlichkeit - Ein Forschungsüberblick unter Anwendungsperspektive, in: HANS P. KRINGS, Wissenschaftliche Grundlagen der Technischen Kommunikation. Tübingen 1996, 129 ff., 137 sowie BERND ULRICH BIERE, Verständlichkeit beim Gebrauch von Fachsprachen, in HOFFMANN/KALVERKÄMPER/WIEGAND, Fachsprachen (Fn. 1), 402 ff., 403.

${ }^{26}$ HARTWIG KALVERKÄMPER, Verständlichkeit, Verständnis und Verständigung im Fadenkreuz: Der Wissenschaftstransfer, in: Kodikas/Code. Ars Semeiotica 11 (1988), $311 \mathrm{ff}$. 316. 
und dabei wiederum intradisziplinär und interdisziplinär, ein andermal als fachextern eingestuft sind (siehe Abb. 2).

\section{Adressatenkreis - Gleitender Übergang von der Fachsprache zur Gemeinsprache}

$\left.\begin{array}{l}\square \text { Fachintern: } \\ \text { - Intradisziplinär (FM/Dis } 1 \rightarrow \text { FM/Dis1) = A1 } \\ \text { - Interdisziplinär (FM/Dis1 } \rightarrow \text { FM/Dis2) = A2 } \\ \square \text { Fachextern }(F M \rightarrow \text { Laie })=A 2\end{array}\right\}$

Abb. 2: Adressatenkreis - Gleitender Übergang von der Fachsprache zur Gemeinsprache

Für die Fach(sprach)lichkeit der untersuchten Rechtstexte wurden die in der Fachsprachenforschung allgemein anerkannten Modelle der vertikalen Schichtung berücksichtigt (z.B. Hoffmann ${ }^{27}$, von Hahn ${ }^{28}$ sowie das besonders auf die vertikale Gliederung der Rechtssprache bezogene Modell von $\mathrm{Otto}^{29}$ ).

\section{Vertikale Gliederung der Rechtssprache (Otto 1981)}

- Gesetzessprache

- Urteils-/Bescheidsprache

- Wissenschafts-/Gutachtensprache

- Behördensprache

- Verwaltungsjargon

- Sonstige

Abb. 3: Ottos Modell der vertikalen Gliederung der Rechtssprache

27 Zur vertikalen Schichtung der Fachsprache vgl. LOTHAR HOFFMANN, Kommunikationsmittel Fachsprache. Eine Einfuihrung. Berlin 1976, 185.

${ }^{28}$ Zur vertikalen Gliederung der Fachsprache vgl. WALTHER vON HAHN, Fachkommunikation. Entwicklung - Linguistische Konzepte - Betriebliche Beispiele. Berlin 1983, 76.

${ }^{29}$ WALTER OTTO, Die Paradoxie einer Fachsprache, in: RADTKE, Die Sprache des Rechts (Fn. 22), 44 ff., 51. 
Wie man sehen kann, befinden sich bei Otto die Gesetzessprache und Urteilssprache, welche in den hier untersuchten Texten in der Fachkommunikation unter einem Aspekt näher analysiert werden, weit oben auf einer Skala abnehmender Fach(sprach)lichkeit.

Urteile wenden sich ja bekanntlich sowohl an die Fachkollegen der nächsten Instanz (A1) als auch an den Rechtsunterworfenen (A2) als Laien, dem als Vermittler oft ein Rechtsanwalt zur Seite steht.

Was die Gesetze betrifft, so gibt es in der Literatur gegensätzliche Auffassungen darüber, ob solche nun primär für den Durchschnittsbürger geschrieben sind oder nicht. Die erste Auffassung wird damit untermauert, dass vom mündigen Bürger erwartet wird, sich an das (gesetzte) Recht zu halten. Das ist nicht so einfach, wenn die sprachlichen Formulierungen des Gesetzes so sind, dass man sie nicht verstehen kann, wie schon Friedrich der $\mathrm{Große}^{30}$ kritisiert hat und was bei Jhering $\mathrm{zu}$ folgendem Diktum geführt hat: „Der Gesetzgeber soll denken wie ein Philosoph, aber reden wie ein Bauer." Vertreter der entgegengesetzten Auffassung, wie Kelsen ${ }^{31}$, sehen jedoch als unmittelbare Adressaten nur die gesetzgebenden Organe bzw. die Vollziehungsorgane, weil diese ermächtigt sind, bei Normverstößen Sanktionen anzuordnen und zu vollstrecken. Diese Auffassung wird auch durch das Diktum des lex iubeat non doceat unterstützt.

Für beide Textsorten wurden folgende zwei Annäherungsweisen gewählt, um zu zeigen, wie ein besseres Verstehen in der Kommunikation bei Mehrfachadressierung ermöglicht werden kann:

Erstens: Das sprachliche Gefälle - der unterschiedliche ,Kode' zwischen Fachmann und Laien - muss überbrückt werden. Wie das möglich ist, geht aus der Untersuchung darüber hervor, wie und wo die Termini in den analysierten Rechtstexten durch Paraphrasen ersetzt werden. Die Platzierung der Paraphrasen auf der heruntergehenden Zugbrücke in Abb. 4 soll diese Vorgehensweise veranschaulichen.

Für diese Vorgehensweise war eine terminologische Begriffsanalyse erforderlich, wobei das Problem der Feststellung, ob ein ,Terminus' vorliegt, in der Terminologielehre als bekanntes Problem gilt. Da ,Terminus', wie dargelegt, als untrennbare Einheit von Begriff und Benennung als Element einer Terminologie gesehen wird, war zu klären, was ein , Begriff' ist.

\footnotetext{
30 Allerhöchste Königl. Cabinets-Order die Verbesserung des Justiz-Wesens betreffend De Dato Potsdam, den 14. April 1780, in: Novum corpus constitutionum Prussico-Brandenburgensium praecipue Marchicarum (NCC), Bd. 6, Sp. 1935, Sp. 1940.

${ }^{31}$ KELSEN, Allgemeine Theorie der Normen (Fn. 12), $40 \mathrm{f}$.
} 


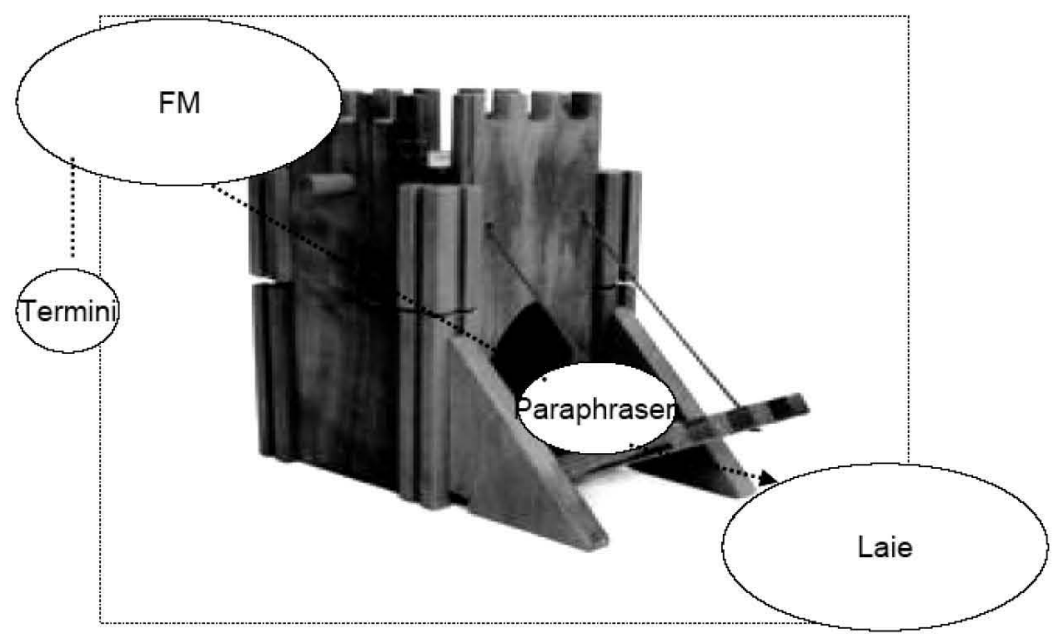

Abb. 4: Sprachliches Gefälle zwischen Fachmann und Laien

Im Gegensatz zu Definitionen, nach denen ,Begriff' als Denkeinheit ${ }^{32}$ gesehen wird, so sehen andere Vertreter ,Begriff' als Wissenseinheit ${ }^{33}$ oder auch als Erkenntniseinheit ${ }^{34}$. Wenn es möglich sein soll, über Begriffe zu kommunizieren, liegt es meines Erachtens auf der Hand, dass die Definition von Denkeinheit entfallen sollte, da Denken immer subjektiv ist und Denkeinheiten daher von Person zu Person unterschiedlich sind. Übrig bleiben dann noch die zwei anderen Definitionen, und zwar ,Begriff' als Wissens- bzw. Erkenntniseinheit zu sehen. ,Begriff' wurde in dieser Untersuchung daher als Wissenseinheit gesehen, in der die zum jeweiligen Zeitpunkt im betreffenden Fachbereich zutreffenden Aussagen über den gegebenen Gegenstand enthalten sind. Somit ist auch die Möglichkeit gegeben, dass sich Begriffe je nach Erkenntnisstand in der Wissenschaft wandeln können, wie beispielsweise beim (strafrechtlichen) Begriff der ,Gewalt'. ${ }^{35}$

32 DIN 2342, (Fn. 13), 1.

33 ISO 1087, (Fn. 20), 2.

34 ÖNORM A 2704: Terminologie. Allgemeine Grundsätze fïr Begriffe und Bezeichnungen. Wien 1990, 3 .

${ }^{35}$ Vgl. hierzu BVerfGE 92, 1 ff., 15; KARIN LUTTERMANN, Gesetzesinterpretation durch Juristen und Laien: Ein rechtslinguistischer Beitrag zum Nötigungstatbestand, in: Linguistische Berichte. Forschung, Information, Diskussion 186 (2001), 157 ff., 162 f.; RALPH CHRISTENSEN/MICHAEL SOKOLOWSKI, Die Bedeutung von Gewalt und die Gewalt von Bedeutung, in: FRIEDRICH MÜLLER/RAINER WIMMER, Neue Studien zur Rechtslinguistik. Berlin 2001, 203 ff., 212. 
Was die ,Rechtsbegriffe' betrifft, ist die Definition mit Rückgriff auf die Definition von Engisch ${ }^{36}$ erfolgt, wonach jeder Begriff, der in einem gültigen Rechtssatz verwendet wird, ohne weiteres als ,Rechtsbegriff' bezeichnet werden kann, wobei der Begriff als Wissenseinheit gesehen wurde.

Nach der Begriffsanalyse war die Beziehung der Termini zu ihren Paraphrasen festzustellen, wobei die in der Rechtswissenschaft bekannte Auseinandersetzung über den Unterschied zwischen Rechtsauslegung und Rechtsfortbildung ${ }^{37} \mathrm{zu}$ berücksichtigen war:

"Am Wortlaut einer Norm braucht der Richter aber nicht haltzumachen. Seine Bindung an das Gesetz [...] bedeutet nicht Bindung an dessen Buchstaben mit dem Zwang zu wörtlicher Auslegung, sondern Gebundensein an Sinn und Zweck des Gesetzes. Die Interpretation ist Methode und Weg, auf dem der Richter den Inhalt einer Gesetzesbestimmung unter Berücksichtigung ihrer Einordnung in die gesamte Rechtsordnung erforscht, ohne durch den formalen Wortlaut des Gesetzes begrenzt zu sein [...]." ${ }^{\text {38 }}$

Wichtig ist dabei aber die für den Untersuchungszweck vorgenommene Abgrenzung: ,Paraphrase' wurde daraufhin eingegrenzt, dass die Paraphrasen sich nur unidirektional lesen lassen, weil sie sich auf einen konkreten Sachverhalt beziehen, und nicht, wie die Termini - im Sinne der DIN 2342 - eine Abstraktion vieler verschiedener gleichgelagerter Sachverhalte im gesetzlich geregelten Tatbestand abdecken.

Zweitens: Das Wissensgefälle zwischen Fachmann und Laien muss ebenfalls überbrückt werden. Dem Fachmann als Träger des Fachwissens, das als Wissen über Begriffe, Begriffsbeziehungen und anzuwendendes domänenspezifisches Methodenwissen gesehen wird, steht der Laie gegenüber ohne dieses Wissensnetz.

Kommunikationstheoretisch gesehen können hier die Termini als Störfaktor bzw. „Lärm” gesehen werden. Diesen Störfaktor gilt es zu beseitigen, wozu der an dieser Untersuchung indirekt am Kommunikationsvorgang beteiligte Interpret mit einbezogen wurde. Der Interpret, $d$. h. ich als diejenige, die ich die Rechtstexte analysiert habe, setzt die Termini - im Sinne der DIN 2342 - zu ihren Begriffssystemen in Beziehung. Auf diese Weise lässt sich dem Laien in der Analyse ein Überblick über den Platz des betreffenden Begriffs im Begriffssystem ver-

\footnotetext{
${ }^{36}$ KARL ENGISCH, Die Relativität der Rechtsbegriffe, in: Deutsche Landesreferate zum 5. Internationalen Kongress für Rechtsvergleichtung. Berlin 1958, 59 ff., 59.

${ }^{37}$ Vgl. RAINER HEGENBARTH, Juristische Hermeneutik und linguistische Pragmatik. Dargestellt am Beispiel der Lehre vom Wortlaut als Grenze der Auslegung. Königstein im Taunus 1982, 19.

${ }^{38}$ BVerfGE 35, 263 ff., 278.
} 
mitteln. Die Platzierung der Begriffssysteme und des Interpreten auf der heruntergehenden Zugbrücke in Abb. 5 soll dies veranschaulichen.

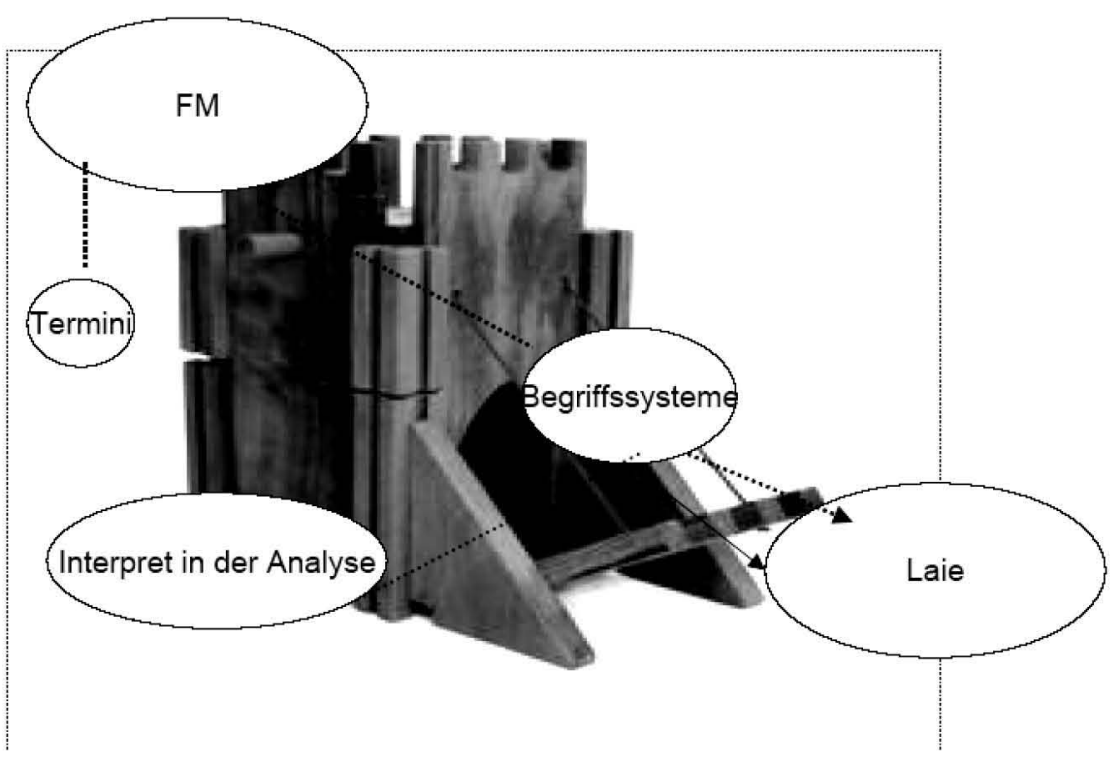

Abb. 5: Wissensgefälle zwischen Fachmann und Laien

Für das Feststellen, wo der jeweilige Begriff seinen Platz im Begriffssystem hat, wurden erneut die Grundsätze der Terminologielehre zugrunde gelegt. Besonders generische Beziehungen, also Beziehungen zwischen Oberbegriff und seinen Unterbegriffen, nehmen dabei einen zentralen Stellenwert ein.

Der oben geschilderte Ablauf kann somit im folgenden zusammenfassenden Modell (Abb. 6) festgehalten werden.

Das vereinfachte Kommunikationsmodell aus Abb. 1 ist hier um die verschiedenen Aspekte, die erwähnt wurden, ausgebaut worden. Sender, Adressat und ",angekommene" Botschaft sind jeweils konkretisiert worden als Richter, A1 bzw. A2 und Urteil. Die dunkel unterlegten "Wolken" sollen veranschaulichen, wie beim Sender dessen Fachwissen, Weltwissen und episodisches Wissen ("top-down") in das Enkodieren mit einfließen, während der Rechtsunterworfene als A2 beim Dekodieren („,bottom-up") dort ggf. Lücken hat. 


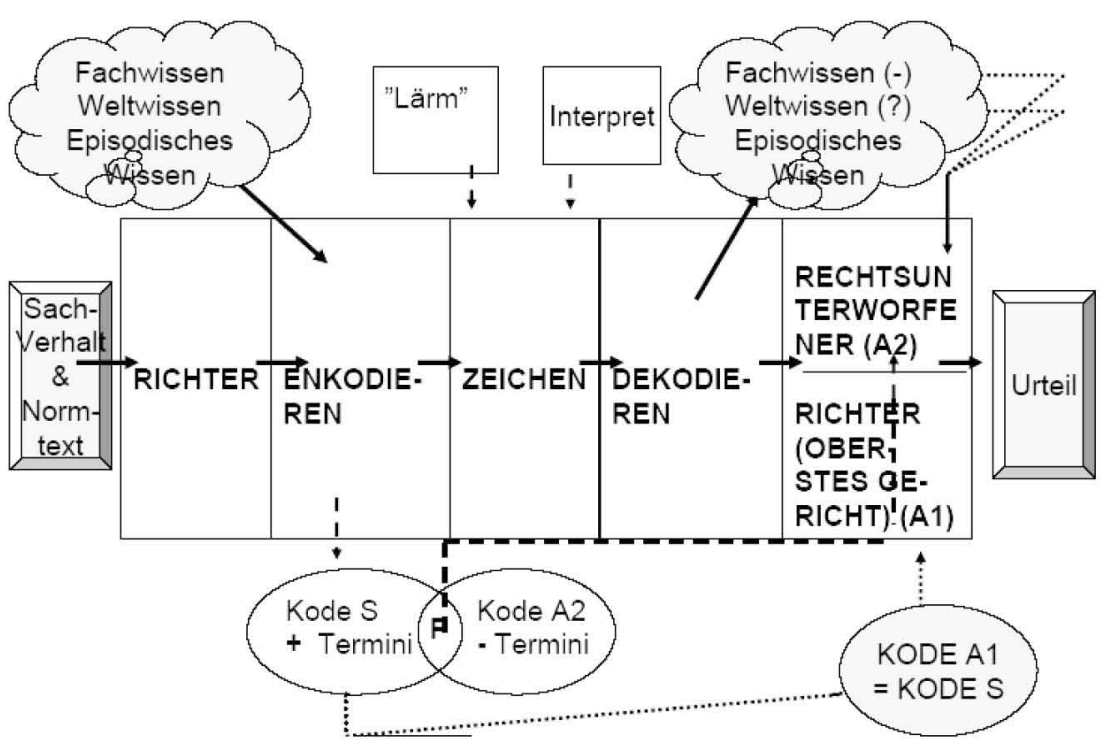

Abb. 6: Zusammenfassendes Modell

Das dunkel unterlegte Kästchen auf der linken Seite des Modells veranschaulicht die Grundlagen, die der Sender bei der Abfassung seiner intendierten Botschaft $\mathrm{zu}$ beachten hat. Beim A2 darf der Sachverhalt als bekannt vorausgesetzt werden, weil diese Person ja (normalerweise) weiß, was sie getan hat. Beim A1 (der nächsten Instanz) erschließt sich der Sachverhalt aus der Sachverhaltsbeschreibung im Urteil der unteren Instanz.

Das dunkel unterlegte Kästchen auf der rechten Seite des Modells veranschaulicht die "angekommene" Botschaft, die sich idealiter mit der intendierten Botschaft des Senders deckt.

Die zwei elliptischen Kreise sind gegenüber dem ersten Modell um zwei Eintragungen erweitert worden. Gemeinsam sind den beiden Kodes (im überlappenden Teil) die Paraphrasen. Die gestrichelte Linie von dort $\mathrm{zu}$ "A2" soll verdeutlichen, dass A2 eher die Paraphrasen verstehen kann, während die Termini aus Rücksicht auf A1 (nächste Instanz) zwecks Rechtskontrolle verwendet werden. 


\section{Ergebnis}

In der diesem Beitrag zugrunde liegenden Untersuchung 39 war dem ersten „eigentlichen" Analyseschritt über die Begriffe im Hinblick auf ihre Merkmale ein weiterer Untersuchungsschritt in Form eines Textvergleichs zwischen Tenor und den dazugehörigen Gesetzesstellen vorgeschaltet, um dem Laien in Sachen Recht den Sinninhalt des Textes von der Struktur her durchschaubar(er) zu machen. Durch Anwendung von Begriffsbeziehungen, hier besonders den hierarchischen, und dabei wiederum den Abstraktionsbeziehungen, wurden zwecks eines ersten Überblicks Mikrobegriffssysteme (exemplarisch über die Zueignungsdelikte oder die schuldrechtlichen Verträge) erstellt. Die Begriffe wurden durch ihre Merkmale voneinander abgegrenzt, und da Merkmale wiederum den Status eines Begriffes haben und sich dieser erneut durch spezifische Merkmale von seinem Oberbegriff unterscheidet ... ad infinitum, breitet sich ein weites Netz an extralinguistischem Wissen vor dem Interpreten aus. Dieses Wissen reicht viel weiter, als das, was man üblicherweise mit ,Bedeutung' verbindet bzw. man als ,Bedeutungsfeststellung' bezeichnen kann. Auf diese Weise konnte dieser Analyseschritt sehr deutlich den Unterschied zeigen zwischen der ,Bedeutung', die der Laie kennt, und der ,Bedeutung', die sich dem Fachmann bei Verwendung eines Terminus erschließt. Dies ist an sich nicht unerwartet, da bei einem Fachmann qua Fachmann das genaue(re) Wissen vorausgesetzt werden darf. Wohl aber dürfte sich gezeigt haben, dass das Netz an extralinguistischem Wissen dabei wesentlich weit gespannter ist als im Voraus angenommen.

Es hat sich aber auch zeigen lassen, dass ein Begriff durchaus dynamisch ist, und sogar sein muss, da neue Erkenntnisse und Veränderungen in der Gesellschaft zu einer anderen als der bisherigen Definition führen, die Benennung jedoch unverändert bleibt. Da ,Begriff' als Wissenseinheit gesehen wurde, trifft die Dynamik auch auf das (Fach)Wissen zu, hier besonders als Aneignung des (Fach)Wissens durch den Laien, sogenannte Interiorisierung, die eindeutig als Verstehensvoraussetzung gesehen wurde.

Der nächste Analyseschritt konzentrierte sich auf jene Teile von Urteilen, die 'Sachverhaltsbeschreibung' und ,Urteilsgründe' genannt werden, mit Blick auf die dort vorkommenden Paraphrasen von den im vorherigen Schritt festgestellten Termini. ${ }^{40}$

\footnotetext{
39 SIMONNÆS, Verstehensprobleme bei Fachtexten (Fn. 15).

${ }^{40}$ Für den vollen Wortlaut der dieser Analyse zugrunde liegenden Urteilsakten sei auf SIMONNÆS, Verstehensprobleme bei Fachtexten (Fn. 15), Teil II verwiesen.
} 
Mein Befund ist, dass die Paraphrasen dabei nicht nur neue sprachliche (Re)formulierungen, sondern auch einen Wechsel von der abstrakten Ebene der Begriffe hin auf die ontische Ebene des Einzelfalles darstellen (müssen). Aufgrund der sowohl lexikalisch als auch syntaktisch vollständigen Reformulierung konnte jedoch die Beziehung zu ihren gesetzlichen Termini nur über ein umständliches Verfahren unter Berücksichtigung des linguistischen und extralinguistischen Wissen dargelegt werden. Das extralinguistische Wissen spiegelt dabei die Sachverhaltsbeschreibung wider, die Teil der Entscheidungsgründe ist.

Der letzte Analyseschritt konzentrierte sich darauf, festzustellen, ob und warum in den letztinstanzlichen Urteilen eher Termini als Paraphrasen verwendet werden. Es hat keine zahlenmäßige Erfassung stattgefunden, sondern als Nachweis für die Verwendung von Termini wurden als relevant erachtete Textstellen aus dem letztinstanzlichen Urteil zugrunde gelegt. Dies ist selbstredend ein subjektives Moment, legt aber dennoch das Verfahren zur Feststellung offen.

In den letztinstanzlichen Urteilen (hier des BGH) sind durch die Auslegung des/der entsprechenden Paragraphen neue Termini (exemplarisch ,Revision' und ,Tateinheit') hinzugekommen. Diese Auslegungen sind nicht weiter anfechtbar, und daher ergibt sich eine Kommunikationssituation, in der sich Fachleute gegenüberstehen, die Richter des BGH und Richter anderer nachgeordneter Gerichte, die künftig in ähnlichen Fällen $\mathrm{zu}$ entscheiden haben. Ihnen dienen solche früheren höchstrichterlichen Urteile als Leitsätze. Daher ist es meines Erachtens nur natürlich, dass die Argumentation eher auf begrifflicher als auf ontischer Ebene stattfindet.

\section{Zusammenfassend bleibt Folgendes festzuhalten:}

- Die Hypothese 1 (Doppelstrategie) hat sich bestätigen lassen. Es konnte für die untersuchten Urteile nachgewiesen werden, dass besonders in den Unterinstanzen von der abstrakten Ebene auf die ontische - einzelfallbezogene - Ebene übergewechselt wird, um dem Rechtsunterworfenen einsichtig $\mathrm{zu}$ machen, warum sein Verhalten den Tatbestandsmerkmalen der betreffenden gesetzlichen Regelungen entspricht. Für die Richter an den höheren Instanzen als den anderen angesprochenen und mit Kelsen als den primären Adressatenkreis ist dabei jedoch wichtig, dass die Argumentation zusätzlich auf einer abstrakteren Ebene geführt wird, wie dies besonders in den Teiltexten Urteilsformel als auch Urteilsgründen der Fall ist.

- Die Hypothese 2 (Beitrag zur besseren Verständlichkeit) hängt ebenfalls eng mit der jeweiligen Kommunikationssituation zusammen, nämlich, ob fachinterne oder fachexterne Verwendung der Rechts- 
sprache vorliegt. Es ist versucht worden, nachzuweisen, dass der Gebrauch von Termini eine Verständnisbarriere darstellen kann, sofern der Adressat nicht die dazugehörigen Begriffssysteme kennt. Das Vermitteln von Begriffssystemen ist somit eine Methode zur Verständnissicherung, wobei die grafische Darstellung dem Adressaten einen schnellen Überblick ermöglicht. Die verbale Darlegung eines Begriffssystems ist dagegen viel umständlicher und daher eher für didaktische Zwecke geeignet. In der fachexternen Kommunikationssituation sind erwartungsgemäß Paraphrasen leichter verständlich, wobei allerdings zu beachten ist, dass die Paraphrasen nur unidirektional gelesen werden können. Ebenso müssen die vorgefundenen Paraphrasen als exemplarisch gelten, da jeder Richter aufgrund des vorgegebenen Tatbestandes nach entsprechenden sprachlichen Realisierungen sucht, deren es eine unendliche Vielzahl gibt, zu denen er kraft seiner Kompetenz (sensu Chomsky) ${ }^{41}$ imstande ist. Andere verständniserschwerende Aspekte sind jedoch in dieser Untersuchung ausgeklammert geblieben.

- Das Abnehmen von Paraphrasen in letztinstanzlichen Urteilen (Hypothese 3) hat sich ebenfalls nachweisen lassen. Die Doppelstrategie des Richters der unteren Instanz braucht nicht im selben Maße beachtet zu werden, da letztinstanzliche Urteile nicht weiter anfechtbar sind. Letztinstanzliche Urteile richten sich somit auch an andere Experten der Rechtssprache, besonders Richter, denen die dort getroffenen Entscheidungen als Interpretation von gesetzlichen Tatbeständen künftig als Leitsätze dienen, wenn ähnlich gelagerte Sachverhalte zu beurteilen sind. Mit anderen Worten liegt dann eine fachinterne Kommunikationssituation vor.

\section{Ausblick}

Der Nutzen dieser Analyse wird im Nachweis dessen gesehen, wie sich zentrale Herausforderungen zum besseren Verständnis/Verstehen in der Fachkommunikation im Recht bei Berücksichtigung der Mehrfachadressierung lösen ließen, und zwar

- durch Rückgriff auf Begriffssysteme, wie aus der Terminologielehre bekannt

- durch Paraphrasen, die dabei als gemeinsamer Kode für Sender und beide Adressaten (A1 und A2) gesehen wurden. Voraussetzung dafür, die Paraphrasen verstehen und zu ihren Termini in Beziehung

\footnotetext{
${ }^{41}$ NOAM CHOMSKY, Aspects of the Theory of Syntax. Cambridge (Mass.) 1965, 3.
} 
setzen zu können, ist eine Interpretation (,Auslegung' in der Sprache des Juristen), was wiederum ein entsprechendes, Vorverständnis' voraussetzt.

Andere Faktoren, die ebenso zur besseren Verständlichkeit beitragen können, wie syntaktische Einfachheit, wurden in dieser Arbeit jedoch nicht berücksichtigt.

Verstehensprobleme in der Fachkommunikation bei Mehrfachadressierung wurden in dieser Untersuchung unter intralingualem Aspekt gesehen. Eine Interpretation eines vorgegebenen Textes findet aber auch am Anfang eines interlingualen Übersetzensprozesses statt. Von daher dürfte die beschriebene Herangehensweise ebenfalls für didaktische Zwecke nutzbringend sein.

Zu guter Letzt gilt: Für eine jede Gesellschaft ist es wichtig, dass die Rechtsunterworfenen das Recht verstehen und somit in der Lage sind, das Recht zu befolgen. Begriffssysteme und Interpretation (Auslegung) können dabei eine gute Hilfe sein, wenn der Adressat gewillt ist, sich durch diese Hilfsmittel helfen zu lassen bzw. sich selbst dieser Hilfsmittel zu bedienen. 


\section{Literatur}

Biere, Bernd Ulrich, Verständlichkeit beim Gebrauch von Fachsprachen, in: Lothar Hoffmann/Hartwig Kalverkämper/Herbert Ernst Wiegand (Hrsg.) (Hrsg.), Fachsprachen - Languages for Special Purposes. Ein internationales Handbuch zur Fachsprachenforschung und Terminologiewissenschaft - An International Handbook of Special Languages and Terminology Research. 1. Halbbd. Berlin 1998, 402 ff.

Bungarten, Theo (Hrsg.), Fachsprachentheorie. Bd. 2: Konzeptionen und theoretische Richtungen. Tostedt 1993.

Chomsky, Noam, Aspects of the Theory of Syntax. Cambridge (Mass.) 1965.

Christensen, Ralph/Sokolowski, Michael, Die Bedeutung von Gewalt und die Gewalt von Bedeutung, in: Friedrich Müller/Rainer Wimmer (Hrsg.), Neue Studien zur Rechtslinguistik. Berlin 2001.

Christmann, Ursula/Groeben, Norbert, Textverstehen, Textverständlichkeit Ein Forschungsüberblick unter Anwendungsperspektive, in: Hans P. Krings (Hrsg.), Wissenschaftliche Grundlagen der technischen Kommunikation. Tübingen 1996, $129 \mathrm{ff}$.

DIN 2342 TEIL 1, Begriffe der Terminologielehre. Grundbegriffe. Normenausschuß Terminologie (NAT), Deutsches Institut für Normung e. V. Berlin 1992.

Duve, Hans Ernst/Weirich, Hans-Armin, Die Verständigung zwischen dem Bürger und den Juristen kann verbessert werden, in: Ingulf Radtke (Bearb.), Die Sprache des Rechts und der Verwaltung. Stuttgart 1981, 119 ff.

Engisch, Karl, Die Relativität der Rechtsbegriffe, in: Deutsche Landesreferate zum 5. Internationalen Kongress für Rechtsvergleichung. Berlin 1958, 59 ff.

Friedrich II., Allerhöchste Königl. Cabinets-Order die Verbesserung des Justiz-Wesens betreffend. De Dato Potsdam, den 14. April 1780, in: Novum Corpus Constitutionum Prussico-Brandenburgensium praecipue Marchicarum (NCC). Herausgegeben von Christian Otto Mylius. Berlin 1753-1822. Bd. 6, Sp. 1935-1944.

Gadamer, Hans-Georg, Vom Zirkel des Verstehens, in: Günther Neske (Hrsg.), Martin Heidegger zum siebzigsten Geburtstag. Festschrift. Pfullingen 1959, $24 \mathrm{ff}$.

Gülich, Elisabeth/Raible, Wolfgang, Textsorten-Probleme, in: Hugo Moser/ Hans Eggers/Johannes Erben/Odo Leys/Hans Neumann (Hrsg.), Linguistische Probleme der Textanalyse. Jahrbuch 1973. Düsseldorf 1975, $144 \mathrm{ff}$.

Haas, Rudolf, Recht und Sprache, in: Rudolf Haas (Hrsg.), Recht und Sprache. Festschrift zum 10jährigen Bestehen der Deutschen Anwaltsakademie. München $1989,39 \mathrm{ff}$.

Hahn, Walther von, Fachkommunikation. Entwicklung - Linguistische Konzepte - Betriebliche Beispiele. Berlin 1983. 
Helbig, Gerhard, Entwicklung der Sprachwissenschaft seit 1970. 2. Aufl., Leipzig 1986.

Hegenbarth, Rainer, Juristische Hermeneutik und linguistische Pragmatik. Dargestellt am Beispiel der Lehre vom Wortlaut als Grenze der Auslegung. Königstein im Taunus 1982.

Hoffmann, Lothar, Kommunikationsmittel Fachsprache. Eine Einführung. Berlin 1976.

Hoffmann, Lothar, Fachwissen und Fachkommunikation. Zur Dialektik von Systematik und Linearität in den Fachsprachen, in: Theo Bungarten, Fachsprachentheorie. Bd. 2: Konzeptionen und theoretische Richtungen. Tostedt $1993,595 \mathrm{ff}$.

Hoffmann, Lothar/Kalverkämper, Hartwig/Wiegand, Herbert Ernst (Hrsg.), Fachsprachen - Languages for Special Purposes. Ein internationales Handbuch zur Fachsprachenforschung und Terminologiewissenschaft - An International Handbook of Special Languages and Terminology Research. 1. Halbbd. Berlin 1998.

ISO 1087-1, Terminology work - Vocabulary. Theory and Application. 2000.

Jayme, Erik (Hrsg.), Langue et Droit. XVe Congrès International de Droit Comparé, Bristol 1998, Collection des rapports. Brüssel 1999.

Kalverkämper, Hartwig, Fachexterne Kommunikation als Maßstab einer Fachsprachen-Hermeneutik: Verständlichkeit kernphysikalischer Fakten in spanischen Zeitungstexten, in: Ders. (Hrsg.), Fachsprachen in der Romania. Tübingen $1988,151 \mathrm{ff}$.

Kalverkämper, Hartwig, Verständlichkeit, Verständnis und Verständigung im Fadenkreuz: Der Wissenschaftstransfer, in: Kodikas/Code. Ars Semeiotica 11 (1988), $311 \mathrm{ff}$.

Kelsen, Hans, Allgemeine Theorie der Normen. Wien 1979.

Klein, Wolfgang, Sprache des Rechts. Vermitteln, Verstehen, Verwechseln, in: Zeitschrift für Literaturwissenschaft und Linguistik 118 (2000), $7 \mathrm{ff}$.

Kühn, Peter, Juristische Fachtexte, in: Gerhard Helbig/Lutz Götze/Gert Henrici/Hans-Jürgen Krumm (Hrsg.), Deutsch als Fremdsprache. Ein internationales Handbuch. 1. Halbbd. Berlin 2001, $582 \mathrm{ff}$.

Lasswell, Harold D., The Structure and Function of Communication in Society, in: Lyman Bryson (Hrsg.), The Communication of Ideas. A series of addresses, New York 1948, $37 \mathrm{ff}$.

Laurén, Christer/Myking, Johan/Picht, Heribert, Terminologie unter der Lupe. Wien 1998

Luttermann, Karin, Gesetzesinterpretation durch Juristen und Laien: Ein rechtslinguistischer Beitrag zum Nötigungstatbestand, in: Linguistische Berichte. Forschung, Information, Diskussion 186 (2001), $157 \mathrm{ff}$.

Martin, Robert, Inférence, Antonymie et Paraphrase, Eléments pour une théorie sémantique. Paris 1976. 
Novalis, Novalis' Werke. Dritter Teil. Fragmente 1. Herausgegeben von Hermann Friedemann. Berlin 1921.

ÖNORM A 2704, Terminologie. Allgemeine Grundsätze für Begriffe und Bezeichnungen. Wien 1990.

Otto, Walter, Die Paradoxie einer Fachsprache, in: Ingulf Radtke (Bearb.), Die Sprache des Rechts und der Verwaltung. Stuttgart 1981, $44 \mathrm{ff}$.

Picht, Heribert, Fachkommunikation - Fachsprache, in: Gerhard Budin (Hrsg.), Multilingualism in specialist communication: Proceedings of the 10th European LSP Symposium, Vienna, 29. Aug.-1. Sept. 1995. Vol. 1. Wien 1996, $27 \mathrm{ff}$.

Schellhammer, Kurt, Die Arbeitsmethode des Zivilrichters. 12. Aufl., Heidelberg 1997.

Simonnæs, Ingrid, Zur Hermeneutik als Verstehenshilfe bei Rechtstexten, in: LSP \& Professional Communication. An International Journal (Formerly Unesco Alsed-LSP Newsletter) 1 (2001), $78 \mathrm{ff}$.

Simonnæs, Ingrid, Verstehensprobleme bei Fachtexten. Zu Begriffssystemen und Paraphrasen als Visualisierungs- bzw. Verbalisierungsinstrumente in der Kommunikation zwischen Fachmann und Laien. Eine Untersuchung anhand gerichtlicher Entscheidungen. Diss. Bergen 2003.

Schwarz, Monika, Einführung in die kognitive Linguistik. 2. Aufl., Tübingen 1996.

Vanderlinden, Jacques, Langue et Droit, in: Erik Jayme (Hrsg.), Langue et Droit. XVe Congrès International de Droit Comparé, Bristol 1998, Collection des rapports. Brüssel 1999, $65 \mathrm{ff}$.

Warnke, Ingo, Transferwissenschaftliche Aspekte der Schwerverständlichkeit deutscher Gesetzestexte, in: Sigurd Wichter/Gerd Antos (Hrsg.), Wissenstransfer zwischen Experten und Laien. Umriss einer Transferwissenschaft. Frankfurt am Main 2001, 193 ff.

Wolski, Werner, Fachtextsorten und andere Textklassen: Probleme ihrer Bestimmung, Abgrenzung und Einteilung, in: Lothar Hoffmann/Hartwig Kalverkämper/Herbert Ernst Wiegand (Hrsg.), Fachsprachen - Languages for Special Purposes. Ein internationales Handbuch zur Fachsprachenforschung und Terminologiewissenschaft - An International Handbook of Special Languages and Terminology Research. 1. Halbbd. Berlin 1998, 457 ff. 